\title{
Telovelar Approach for Midline Posterior Fossa Tumors in Paediatrics: 25 Cases Experience
}

\author{
Mohamed I Refaat ${ }^{\star}$, Ehab A Elrefaee and Walid E Elhalaby
}

Neurosurgery Department, Cairo University, Egypt

*Corresponding author: Mohamed I Refaat, Neurosurgery Department, Cairo University, Egypt, Tel: +201001797978; E-mail: mrefaat_77@yahoo.co.uk Rec date: Nov 01, 2016; Acc date: Nov 15, 2016; Pub date: Nov 20, 2016

Copyright: () 2016 Refaat MI. This is an open-access article distributed under the terms of the Creative Commons Attribution License, which permits unrestricted use, distribution, and reproduction in any medium, provided the original author and source are credited.

\begin{abstract}
Background: The classic transvermian route for excision of fourth ventricular tumors could be associated with postoperative cerebellar mutism. The Telovelar approach leads to the fourth ventricle via the cerebellomedullary fissure, avoiding retraction on the dentate nuclei and vermian incisions, hence lowering the incidence of cerebellar mutism. Objective: The aim of this study is to evaluate the Telovelar approach for excision of fourth ventricular tumors.
\end{abstract}

Patients and methods: This retrospective study was conducted on 25 cases with midline posterior fossa tumors collected between 2012 and 2014. All cases were operated upon via the telovelar approach succeeded by microscopic tumor excision. Follow up data were collected regarding the degree of excision (total, near total, and subtotal), and postoperative complications. Mean follow up period was 5 months.

Results: Age ranged from 2 to 8 years (mean 5.6 years). The most common was medulloblastoma (13 cases), followed by ependymoma (10 cases), and then choroid plexus papilloma ( 2 cases). Gross total excision was achieved in 8 cases $(32 \%)$, near total excision ( $>80 \%$ of tumor volume) in 14 cases $(56 \%)$, and subtotal excision ( $<80 \%$ of tumor volume) in 3 cases $(12 \%)$. We experienced cerebellar mutism in 2 cases ( $8 \%)$ which improved in one case in the long term postoperative follow up. None of the cases had truncal ataxia. Two cases (8\%) had postoperative facial palsy, while 3 cases $(12 \%)$ had postoperative bulbar affection. There were two mortalities in this study, which were related to bulbar palsy and chest infection.

Conclusion: Telovelar approach to the fourth ventricle is becoming a widespread technique for removing midline posterior fossa tumors. It gives excellent access and visualization to the fourth ventricle, with a low incidence of postoperative cerebellar mutism.

Keywords: Fourth ventricle; Telovelar; Cerebellar mutism; Medulloblastoma; Ependymoma

\section{Background}

Pediatric brain tumors represent $15 \%$ to $20 \%$ of all brain tumors; they are the second most common type of pediatric tumors after leukemia. Posterior fossa tumors are the commonest primary brain tumors in pediatrics. Over two thirds of all brain tumors in children older than 1 year are in the posterior fossa. Common tumors are: pilocytic astrocytomas, ependymomas, medulloblastomas, as well as, brain stem gliomas $[1,2]$.

A child with a posterior fossa tumor usually presents with manifestations of hydrocephalus and increased intracranial pressure. This typically presents with headache, nausea, vomiting, irritability in younger children, and 6th nerve palsy. Large sized tumors can present with long tract manifestations, facial palsy, bulbar palsy, as well as ataxia and hypotonia $[1,3,4]$.

Management of fourth ventricular tumors usually starts with management of hydrocephalus if present. Variable options include external ventricular drainage, endoscopic third ventriculostomy, or ventriculo-peritoneal shunting. Direct surgical tumor excision, via a midline suboccipital craniotomy, then follows. Two routes can give access to the fourth ventricle, either via a vermian incision, or via the cerebello-medullary fissure (Telovelar approach). The transvermian route is suspicious of causing the postoperative complication of cerebellar mutism $[5,6]$.

Cerebellar mutism is a common postoperative complication following surgical resection of fourth ventricular tumors. It usually starts with reduction in speech output, progressing to mutism. It's usually associated with hypotonia, emotional liability, irritability and ataxia. Usually cerebellar mutism subsides with few months after surgery, yet in some cases it can persist up to a year postoperatively [7]. The exact etiology of cerebellar mutism remains unclear. It's suspected to be caused by compression on both dentate nuclei, injuring the neuronal tracts running from the dentate nuclei through the ventrolateral thalamus to the supplementary motor cortex. The incidence increases with large sized tumors, medulloblastomas, as well as in younger children [8]. Gaining access to the tumors via the telovelar approach can decrease the possibility of cerebellar mutism as it minimizes the retraction on the dentate nuclei.

\section{Patients and Methods}

This is a retrospective study conducted on 25 successive cases presenting with midline posterior fossa tumors related to the fourth ventricle. Children at any age less than 12 years were included in this 
study. Vermian, cerebellar, and brain stem tumors were not included in this study. All other forms of fourth ventricular tumors were included. The study was conducted between 2012 and 2014 in the pediatric section of the Neurosurgery department, Cairo University. The follow up period ranged from 1-18 months (mean 5 months).

A full clinical history was taken for the cases. Full preoperative and postoperative neurological examination was also done. Routine preoperative laboratory investigations, contrast enhanced CT and MRI brain were done for all cases preoperatively.

\section{Surgical approach}

All 25 cases were managed initially for hydrocephalus by insertion of ventriculoperitoneal shunt few days before surgery (three to seven days). The telovelar approach was planned for all cases. Preoperative cross matched blood was prepared for all cases. Standard Neuroanesthesia were applied including invasive arterial blood pressure monitoring, non-invasive $\mathrm{CO} 2$ monitoring, positive end expiratory pressure monitoring. Patients were positioned in prone position. A midline suboccipital craniectomy is done extending to the foramen magnum (sometimes included posterior arch of atlas), followed by Y shaped dural incision. Microscopic excision of the tumor then follows. Usually large tumors present between the two cerebellar tonsils, stretching the arachnoid overlying the craniocervical junction and the inferior medullary velum. If not, opening the inferior medullary velum will open the caudal end of the fourth ventricle, exposing the inferior part of the tumor. The tumor is then resected from the deep part of the fourth ventricle, extending cranially up to the aqueduct, and laterally to the foramina of Luschka. Tumors invading the brain stem are reduced as much as possible to the level of the normal surrounding floor. Haemostasis is then obtained, trying as much as possible to reduce the use of haemostatic agents, as they can be misinterpreted as residual tumor in the follow up images. Closure of layers then follows.

Immediate post-operative clinical and radiological follow-up was performed for all cases. The tumor is sent for histopathologic examination.

The data collected from the 25 cases were analyzed for clinical presentation, tumors' histopathology, degree of tumor removal, and postoperative complications. The extent of tumor removal was divided to three main categories:

1) Gross total excision (100\% of tumor volume is removed).

2) Near total excision (more than $80 \%$ of tumor volume is removed).

3) Subtotal excision (less than $80 \%$ of tumor volume is removed).

\section{Results}

\section{Patient population}

There were 14 (56\%) females and 11 males (44\%), their age ranged from 2 to 8 years; the mean age in our study was 5.6 years.

\section{Clinical picture}

21 of our 25 cases $(84 \%)$ presented to us with manifestations of hydrocephalus and increased intracranial pressure. Among these 21 cases; 7 cases presented with persistent headache only. 9 cases had the classic persistent vomiting, headache, nausea, with blurring of vision, in 2 of these cases this classic picture was associated with sixth nerve palsy. 3 cases presented with physical growth retardation and persistent vomiting. While 2 cases presented with disturbed conscious level. The remaining 4 cases (16\%) presented with tumor mass effect. 2 cases presented with ataxia and hypotonia, while 2 other cases presented with facial and lower cranial nerve palsies. Headache was the most common presenting symptom, it was found in all 25 cases in variable degrees. The duration of symptoms ranged from 2 days to 3 months (mean 2 weeks).

Anatomically all tumors included in this study were midline fourth ventricular tumors. Their size ranged from $3 \mathrm{~cm}$ to $8 \mathrm{~cm}$ (size of maximal diameter), mean maximal diameter was $5.2 \mathrm{~cm}$. In 9 cases (44\%) the tumor was confined to the boundaries of the fourth ventricle, in 10 cases the tumor extended caudally up to the foramen magnum, in 4 cases the tumor extended cranially till the level of the tentorium, while in 2 cases the tumors extended mainly laterally. Radiologically all our cases showed obstructive hydrocephalus preoperatively, which was primarily managed with insertion of ventriculoperitoneal shunt before tumor excision. (In some cases the shunt will appear in the preoperative MRI images). We didn't meet any cases of upward herniation in this study.

All cases were operated upon via the suboccipital craniectomy, followed by microscopic dissection of the tumors via the telovelar approach. None of our cases had intraoperative difficulties, apart from bleeding and blood transfusion. Postoperative histopathologic examination revealed: 1) medulloblastoma (desmoplastic/nodular type) in 13 cases, 2) ependymoma (WHO grade II) in 10 cases, 3) Choroid plexus papilloma in 2 cases.

\section{Tumor removal}

The extent of tumor removal was determined in each by analysis of the postoperative imaging studies, calculating the percentage of tumor volume left behind. We had gross total excision in 8 cases, near total excision in 14 cases, and subtotal excision in 3 cases (Table 1).

\begin{tabular}{|l|l|l|l|l|}
\hline & $\begin{array}{l}\text { Gross total } \\
\text { excision }\end{array}$ & $\begin{array}{l}\text { Near total } \\
\text { excision }\end{array}$ & $\begin{array}{l}\text { Subtotal } \\
\text { excision }\end{array}$ & Total \\
\hline $\begin{array}{l}\text { Medulloblastom } \\
\text { a }\end{array}$ & $4(30 \%)$ & $8(62 \%)$ & $1(8 \%)$ & 13 \\
\hline Ependymoma & $2(20 \%)$ & $6(60 \%)$ & $2(20 \%)$ & 10 \\
\hline $\begin{array}{l}\text { Choroid plexus } \\
\text { papilloma }\end{array}$ & $2(100 \%)$ & 0 & 0 & 2 \\
\hline Total & $8(32 \%)$ & $14(56 \%)$ & $3(12 \%)$ & 25 \\
\hline
\end{tabular}

Table 1: Degree of tumor excision in relation to pathology.

\section{Post-operative course}

There were no intraoperative mortalities. 7 of our cases (28\%) experienced 4 new postoperative neurological findings including: 2 cases had cerebellar mutism; one of them was associated with postoperative hypotonia, 2 cases had facial palsy, 2 cases had bulbar palsy, and 1 case had both. One of the 2 cases with cerebellar mutism improved after 2 months, while the other remained till the end of the follow up period. None of our cases had postoperative truncal ataxia. There were 2 mortalities in this study (8\%), which was related to bulbar palsy and chest infection (Figures 1-4). 
Citation: Refaat MI, Elrefaee EA, Elhalaby WE (2016) Telovelar Approach for Midline Posterior Fossa Tumors in Paediatrics: 25 Cases Experience. J Neurol Disord 4: 315. doi:10.4172/2329-6895.1000315

Page 3 of 5
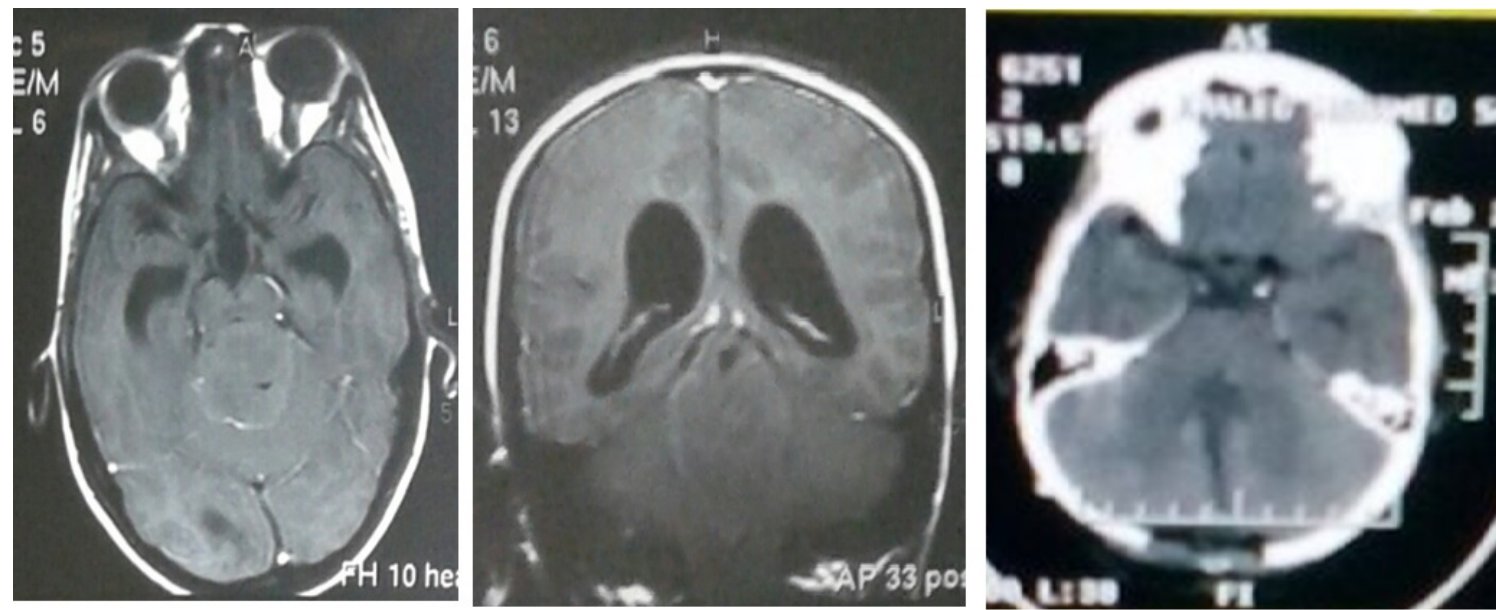

Figure 1: Preoperative MRI of an 8 years old male, presenting with headache and vomiting. Patient was operated upon for tumor excision via telovelar approach. Postoperative CT showed gross total excision. Pathology was: Medulloblastoma. Patient did well with no postoperative complications.
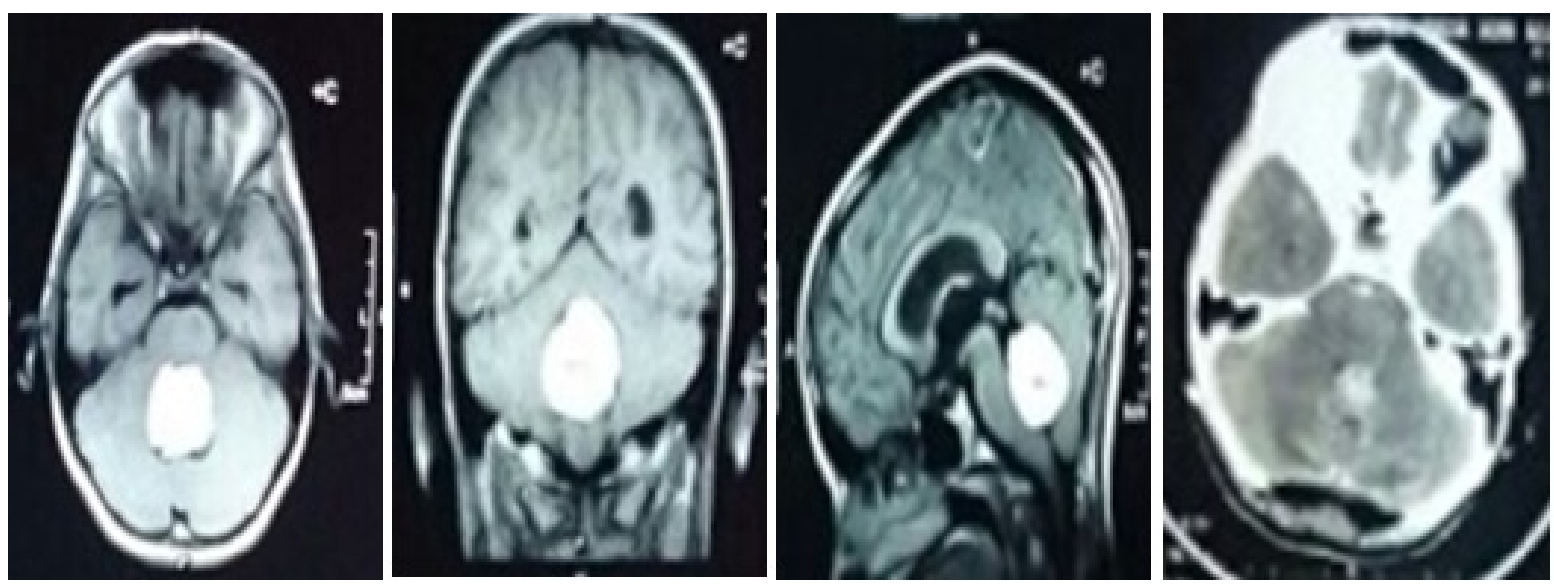

Figure 2: Preoperative MRI of a 7 years old male, with a fourth ventricular tumor. Postoperative CT after tumor excision shows gross total excision. Pathology was choroid plexus papilloma. Patient had no postoperative morbidities.

\section{Discussion}

Tumors of the fourth ventricle remain among the most common tumors in pediatrics. Surgical excision of these tumors represent a challenge to neurosurgeons due to the multiplicity of complications that can result from the surgical technique, and the multiplicity of delicate structures related to the ventricular floor, roof, as well as lower cranial nerves. In the past the conventional route to access the floor of the fourth ventricle was the transvermian approach, by splitting the vermis on the suboccipital surface of the cerebellum [9].

One of the complications following vermian splitting (or retraction) is cerebellar mutism. It is classically formed of decreased speech production or dysarthria, usually progressing to complete mutism. It is associated with hypotonia, ataxia, irritability, and emotional liability. Usually cerebellar mutism is self-limited, but sometimes it can persist up to months following surgery [10].

The telovelar approach was described as an alternative for the classic transvermian route. It gives access to the fourth ventricle via the cerebellomedullary fissure, through opening the tela chorioidea and inferior medullary velum. Large tumors present themselves between the two cerebellar tonsils, stretching the tela chorioidea and inferior medullary velum, which are usually undefined. The telovelar approach avoids vermian incisions and retraction on the dentate nuclei, which is believed to decrease the incidence of cerebellar mutism [11]. 
Citation: Refaat MI, Elrefaee EA, Elhalaby WE (2016) Telovelar Approach for Midline Posterior Fossa Tumors in Paediatrics: 25 Cases Experience. J Neurol Disord 4: 315. doi:10.4172/2329-6895.1000315

Page 4 of 5
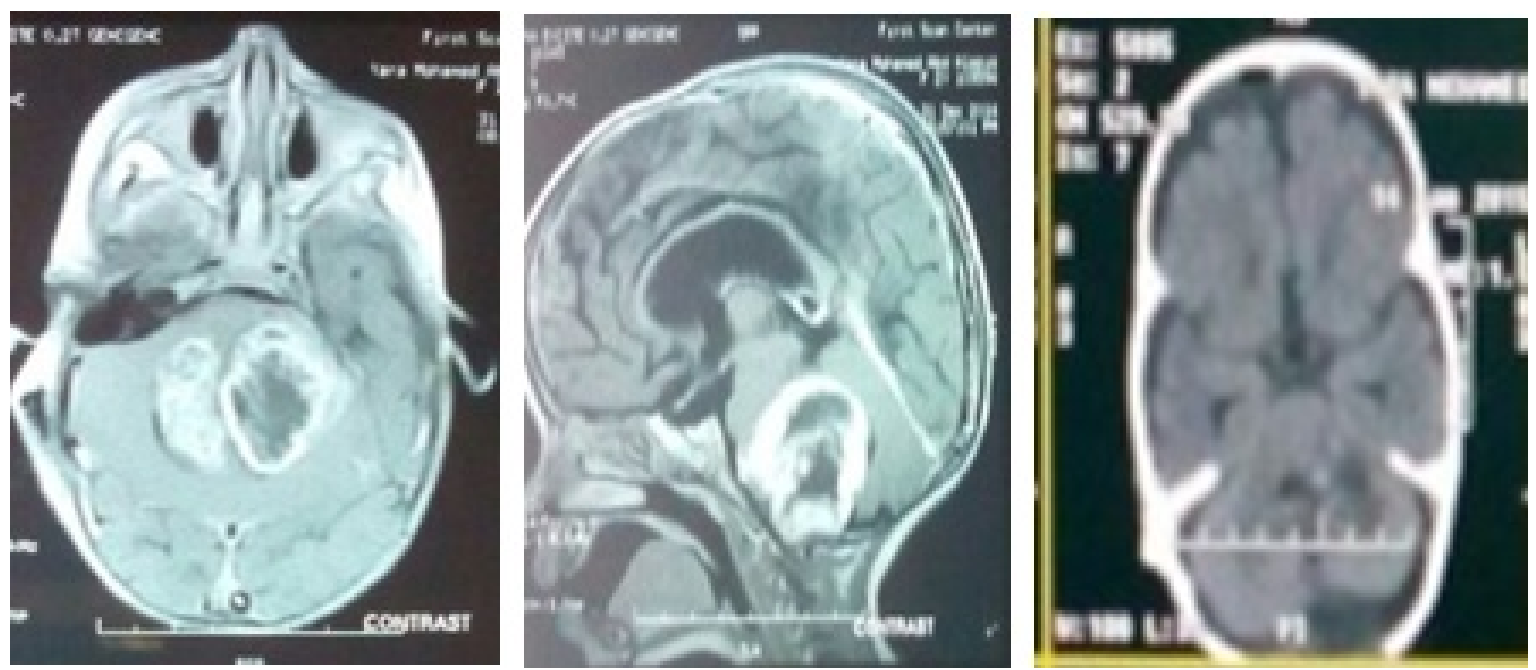

Figure 3: MRI of a 2 years old female, presenting with delayed motor growth and vomiting. Follow up CT showing near total excision of tumor. Pathology was ependymoma. Postoperatively the patient developed bulbar palsy, and she died 2 weeks later from chest infection.
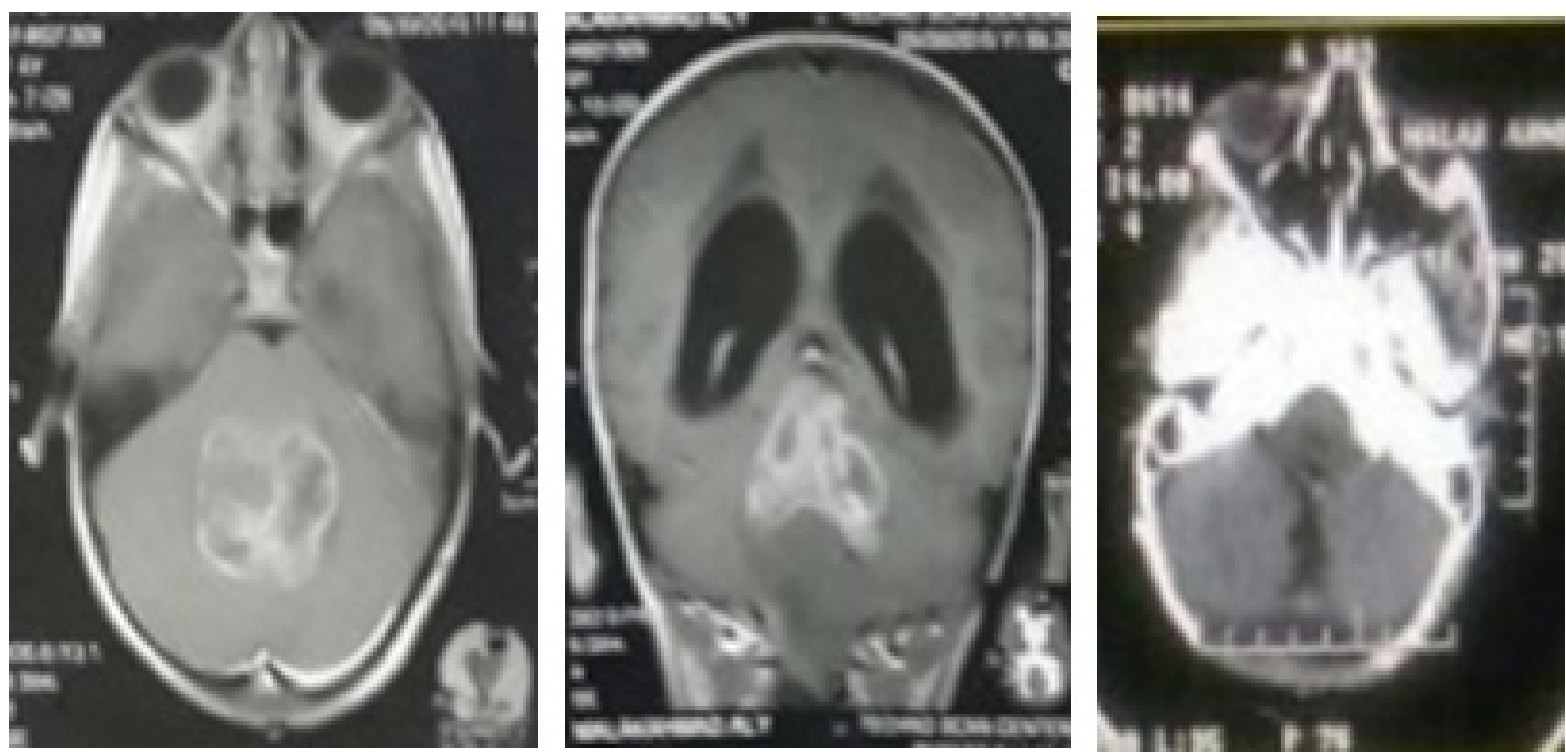

Figure 4: MRI of a 4 years old female presenting with manifestations of hydrocephalus. Post-operative CT showing near total excision of tumor. Pathology was ependymoma. Patient had postoperative cerebellar mutism, which improved after 2 months.

In our study all cases were operated upon via the telovelar approach, data was analyzed for detecting the incidence of postoperative cerebellar mutism. $56 \%$ of cases in this study were females while $44 \%$ were males. Their age ranged from 2 to 8 , years with a mean of 5.6 years. The most common clinical presentation was headache, which was found in all cases. Manifestations of hydrocephalus and increased intracranial pressure were the presenting picture in $84 \%$ of cases $(2$ cases of which $8 \%$ presented with disturbed conscious level). The remaining $16 \%$ presented with tumor mass effect, in the form of ataxia cranial nerve palsies, or hypotonia. The duration of these symptoms ranges from 2 days to 3 months before presenting to us. These facts were coinciding with the literature review concerning epidemiology and clinical picture [1-4].

All cases had initial management for hydrocephalus, by insertion of a medium pressure ventriculoperitoneal shunt in a separate session usually few days before direct tumor attack. We didn't meet any complications with this protocol; none of our patients had manifestations of upward transtentorial herniation. For the 2 cases presenting with altered conscious level, the regained their normal state before direct tumor attack. The surgical approach was the same for all cases. In some cases the tumor was large enough that it presented itself in the cerebellomedullary fissure, pushing both tonsils laterally and stretching the tela and the inferior medullary velum. In such cases no 
retraction was needed. The mean maximal diameter of the tumors in our study was $5.2 \mathrm{~cm}$. Only $44 \%$ of cases tumors were confined to the boundaries of the fourth ventricle, in these cases the inferior medullary velum and the tela bilaterally were resected to gain access to the fourth ventricle. Intermittent use of retraction was needed in some cases, with small sized tumors and for reaching the deep rostral attachment of some tumors. In none of our cases we needed to do a vermian incision to excise the tumor

In this study we had gross total excision in 8 cases (32\%), near total excision in 14 cases (56\%), and subtotal excision in 3 cases (12\%). Histopathologic examination of tumors revealed medulloblastoma in $52 \%$, ependymoma in (40\%), and choroid plexus papilloma in $8 \%$. The highest percentage of total tumor excision was seen in choroid plexus papillomas, followed by medulloblastomas, and then ependymomas. These results were concurring with the results published by El-Bahy, Gok et al., and Tomasello et al. They had percentage of total excision in $69 \%, 86 \%$, and $89 \%$ respectively $[9,11,12]$. (These numbers include our total and near total excisions added together).

We had postoperative mutism in 2 of our cases $(8 \%)$. it could be explained by the fact that most of the tumors in this study opened the way to the ventricle, avoiding vermian incisions and minimizing the use of retraction on the dentate nuclei. This low incidence of cerebellar mutism was also noticed in most published series concerning this issue. El-Bahy [11] and Tomasello et al. [12] didn't have any cases of cerebellar mutism, while Hans et al. [13] had incidence of $8 \%$.

There were 2 mortalities in this study (8\%), which were related to bulbar palsy and pulmonary complications. This was also the same cause of mortalities in the studies published by Tomasello et al. and Hans $[12,13]$.

In our study we found that the main limitation or drawback of the telovelar approach is facing some difficulty in removing the most cranial part of some tumors (the part close to the aqueduct). This part usually needed some retraction to be applied to the cerebellar vermis. This limitation to the telovelar approach was manifested in our results, as in 3 cases $(12 \%)$ we could only achieve a subtotal excision of the tumor.

\section{Conclusion}

Telovelar approach to the fourth ventricle is becoming a widespread technique for removing midline posterior fossa tumors. It gives excellent access and adequate visualization to the fourth ventricle, with early identification of the normal ventricular floor and the brain stem. The use of the telovelar approach prevents vermian incisions and minimizes retraction on the dentate nuclei; this fact decreases the incidence of postoperative cerebellar mutism. The main limitation of the telovelar approach in our experience, was the small high rostral attachment of some tumors, which needed some retraction to be adequately visualized.

\section{References}

1. Aquilina K (2013) Posterior fossa tumors in children- An overview of diagnosis and management. ACNR 13: 24-27.

2. Davis FG, McCarthy BJ (2000) Epidemiology of brain tumors. Curr Opin Neurol 13: 635-640.

3. Massimino M, Giangaspero F, Garrè ML, Gandola L, Poggi G, et al (2011) Childhood medulloblastoma. Crit Rev Oncol Hematol 79: 65-83.

4. Dhall G (2009) Medulloblastoma. J Child Neurol 24: 1418-1430.

5. Gnanalingham KK, Lafuente J, Thompson D, Harkness W, Hayward R (2002) Surgical procedures for posterior fossa tumors in children: does craniotomy lead to fewer complications than craniectomy? J Neurosurg 97: 821-826.

6. Tanriover N, Ulm AJ, Rhoton AL Jr, Yasuda A (2004) Comparison of the transvermian and telovelar approaches to the fourth ventricle. J Neurosurg 101: 484-498.

7. Aquilina $\mathrm{K}$ (2013) The surgical management of posterior fossa tumors in children. ACNR 13: 21-22.

8. Gudrunardottir T, Sehested A, Juhler M, Schmiegelow K (2011) Cerebellar mutism: review of the literature. Childs Nerv Syst 27: 355-363.

9. Gök A, Alptekin M, Erkutlu I (2004) Surgical approach to the fourth ventricle cavity through the cerebello-medullary fissure. Neurosurg Rev 27: 50-54.

10. García Conde M, Martín Viota L, Febles García P, Ravina Cabrera J, López Almaraz R (2007) Severe cerebellar mutism after posterior fossa tumor resection. An Pediatr (Barc) 66: 75-79.

11. El-Bahy K (2005) Telovelar approach to the fourth ventricle: operative findings and results in 16 cases. Acta Neurochir (Wien) 147: 137-142.

12. Tomasello F, Conti A, Cardali S, La Torre D, Angileri FF (2015) Telovelar approach to fourth ventricle tumors: Highlights and limitations. World Neurosurg 83: 1141-1147.

13. Han S, Wang Z, Wang Y, Wu A (2013) Trans-cerebellomedullary fissure approach to lesions of the fourth ventricle: Less is more? Acta Neurochir (Wien) 155: 1011-1016. 